\title{
Cochlear View: Postoperative Radiography for Cochlear Implantation
}

\author{
*Jin Xu, †Shi-Ang Xu, *Lawrence T. Cohen, and *Graeme M. Clark \\ *The Bionic Ear Institute and CRC for Cochlear Implant, Speech and Hearing Aid Innovation, The \\ University of Melboume, Victoria, Australia; and $\dagger$ Cochlear Limited, Sydney, Australia
}

Objective: This study aimed to define a spatial position of the cochlea in the skull based on anatomical studies and to design an appropriate method of skull radiography for demonstration of the multichannel intracochlear electrode array and the structures of the inner ear, for use in evaluating the electrode position and its related pitch perception.

Background: The conventional skull radiograph (plain radiograph) can offer a complete and direct image of an intracochlear electrode array, if the $x$-ray is directed to the cochlea and parallel to the axis of the cochlea.

Methods: Measurement from computed tomography imaging and three-dimensional reconstruction were performed to define the spatial position of the cochlea in the skull.

Results: A radiographic projection, the cochlear view, was designed. A detailed radiographic method and radiologic inter- pretation of the cochlear view is described. An improved clinical method for measuring the longitudinal and angular position of the electrodes from the cochlear view is recommended.

Conclusions: The application of the cochlear view has proved that it is beneficial postoperatively in documenting the results of cochlear implantation, and in evaluating the depth of insertion and position of individual electrodes. It serves as a valuable reference for managing frequency mapping, optimizing speech processing strategies, and further research purposes. The method can be widely used in cochlear implant clinics because of its simplicity, low radiation, speed, and minimal cost. Key Words: Cochlear implant-Cochlear view-Electrode position-Labyrinth-Radiography.

Am J Otol 21:49-56, 2000.
Modern imaging methods, including computed tomography $(\mathrm{CT})$ and magnetic resonance imaging with threedimensional (3D) reconstruction, have dramatically improved imaging of the temporal bone. Today, these are the basic techniques used in preoperative evaluation for cochlear implantation. Conventional radiography, including plain radiographs and polytomography, has no longer been used as a routine preoperative radiologic examination for cochlear implant candidates because it offers little value in providing sufficient detail of the temporal bone (1).

In the last decade (2), postoperative radiologic examination for cochlear implant patients has been required only in cases of unexpected poor performance. Recent advances in multichannel intracochlear implantation have generated interest in correlating individual stimulating electrodes to pitch perception, to develop speech processing schemes that map speech frequencies to appropriate places in the cochlea. Postoperative radiologic

Supported by the Bionic Ear Institute and the Cooperative Research Centre for Speech and Hearing Research, which is funded by the Commonwealth Government of Australia.

Address correspondence and reprint requests to Dr. Jin Xu, Department of Otolaryngology, The University of Melbourne, 32 Gisborne Street, East Melbourne, Vic. 3002, Australia. assessment becomes, therefore, an important procedure. In recent years, cochlear implant researchers have performed investigations designed to document precisely the insertion depth of the electrode array and the location of individual electrodes (3-5). Computed tomography and 3D reconstruction became the focus of this research, because magnetic resonance imaging is contraindicated for postimplantation assessment (6). In our experience, CT is necessary only if complications arise. To establish the position of a multichannel intracochlear electrode, CT is not used routinely, because each tomogram can show only a part of the electrode array. Although a 3D reconstruction of the cochlea can be obtained from a spiral CT scan, images are affected by partial volume averaging, and individual electrodes cannot be clearly distinguished (5). We may expect to get improved CT images in the future as the technology is further improved. At present, conventional skull radiography (plain radiograph) can offer a complete and direct image of the intracochlear electrode array if an appropriate projection is adopted.

To determine a proper view for displaying the intracochlear electrode array, it is necessary to review the corresponding anatomy of the petrous bone, including the cochlea, in relation to the reference lines or planes and other structures of the skull. 
The petrous bone is of a pyramidal shape with its apex pointing medially and anteriorly (Fig. 1). The angle between the axis of the petrous bone and the median sagittal plane of the skull has been reported as varying from
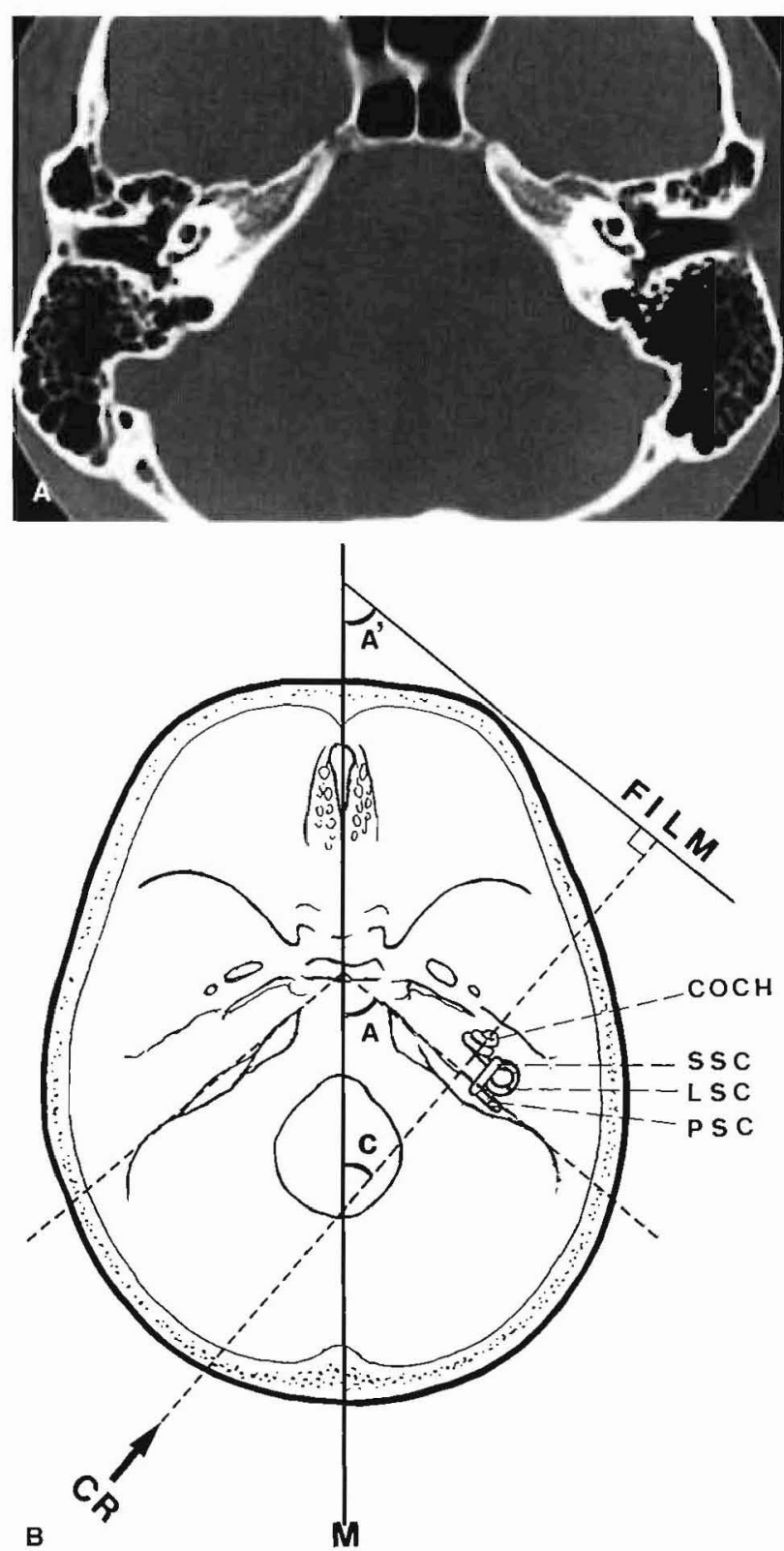

FIG. 1. A: Axial computed tomography section through the cochlea showing the apex of the petrous bone pointing medially and anteriorly, and the cochlea lying in the densest portion of the petrous bone with its apex directing anterolaterally. B: Schematic diagram of the base of the skull viewed from the vertex. Coch $=$ cochlea; SSC = superior semicircular canal; $L S C=$ lateral semicircular canal; $P S C=$ posterior semicircular canal; $C R=$ central $x$-ray beam; $M=$ median sagittal plane of the head; $A=$ the angle between the line passing the lower arm of the posterior semicircular canal and median sagittal plane; $A^{\prime}=$ the angle between the image receptor and the midsagittal plane; $\mathrm{C}=$ the complementary angle between the axis of the cochlea and the median sagittal plane. $40^{\circ}$ to $54^{\circ}(7,8)$. However, the details of these measurements were not reported or cited and the axis of the petrous bone was not specified.

In the densest portion of the petrous bone lies the inner eat (labyrinth), consisting of the vestibule, the semicircular canals, and the cochlea. There tends to be a right angle between the semicircular canals (see Fig. 1 B), although they exhibit some torsion (9). The superior semicircular canal (SSC) is approximately perpendicular to the posterior wall of the petrous bone $(7,8)$. The cochlea is of a spiral shape, approximately two and a half turns around the axis, the modiolus. Its base is at the fundus of the internal auditory meatus (IAM), and its apex is directed anterolaterally (see Fig. 1). The axis of the cochlea and the plane of the SSC as being roughly parallel (see Fig. I B). To our knowledge, there have been no reports in the literature describing the exact direction of the axis of the cochlea in the skull.

Knowledge of the direction of the cochlea in the skull is important in defining the x-ray projection. An ideal picture of the intracochlear electrode array, which is typically located in the basal and middle turns around the modiolus of the cochlea, can be obtained if the $x$-ray is directed at the cochlea and parallel to the modiolus (see Fig. I B).

The major objectives of this study were to define the spatial position of the cochlea in the skull based on anatomical studies, and to design an appropriate method of skull radiography to clearly show the multichannel intracochlear electrode array and the corresponding structures of the inner ear, for evaluating the electrode position and correlating individual stimulating electrodes to pitch perception.

\section{MATERIALS AND METHODS}

\section{Measurements from $\mathrm{CT}$ images in the axial plane}

Thin-section high-resolution CT images on the axial plane were collected from 102 patients ranging in age from 1 to 78 years. The images were processed on bone algorithm. The patients were grouped by age into children ( $1-17$ years) and adults ( $>17$ years). The angle A (see Fig. 1 B) between the line passing through the lower arm of the posterior semicircular canal and the median sagittal line was measured on the image for each patient. This angle $\mathrm{A}$ is regarded as the complementary angle of angle $C$ between the axis of the cochlea and the median sagittal line (see Fig. I B). Determining angle $A$ is important because it represents the angle $A^{\prime}$ between the image receptor and the median sagittal plane of the head (see Fig. I B).

On the left side of each CT image, there is a reading showing an interval between this section and the 0 plane of scanning The readings of the section containing the cochlea (the modiolus section) and the section containing the orifice of the exter nal auditory meatus were recorded from the axial CT images of 50 patients, ranging in age from 1 to 81 years. The difference between these two readings for each patient was calculated, to show the location of the cochlea in relation to the horizonlal plane in the skull. 


\section{Measurements from 3D reconstructed image from histologic sections of temporal bones and Silastic mold of the labyrinth in the vertical plane}

Three human temporal bones were trimmed, decalcified ( $10 \%$ ethylenediaminetetra-acetic acid in neutral buffered formalin), embedded in resin (Spurr) and sectioned at a thickness of $3 \mathrm{\mu m}$. Sections every $130 \mu \mathrm{m}$ were collected and stained with hematoxylin and eosin. The sections were viewed through a microscope, and the image was recorded by a video camera (Panasonic WV-BL200; Matsushita Communication Industrial Co.. Japan) and digitized with a Data Translation DT285I (Data Translation, Inc., Marlboro, MA) frame-grabber card. The outline of the structures of the inner ear was marked manually with a screen cursor or automatically by use of an edge detection algorithm based on gray levels and stored on a personal computer (10). A computer-aided 3D graphic reconstruclion of each inner ear was made (Fig. 2, above). In rotating the reconstructed image to the direction when viewing from lateral side of the labyrinth (see Fig. 2, below), the angle (B) between the lateral semicircular canal (LSC) and the axis of the cochlea was measured.

Seven cadaver adult human temporal bones were used 10

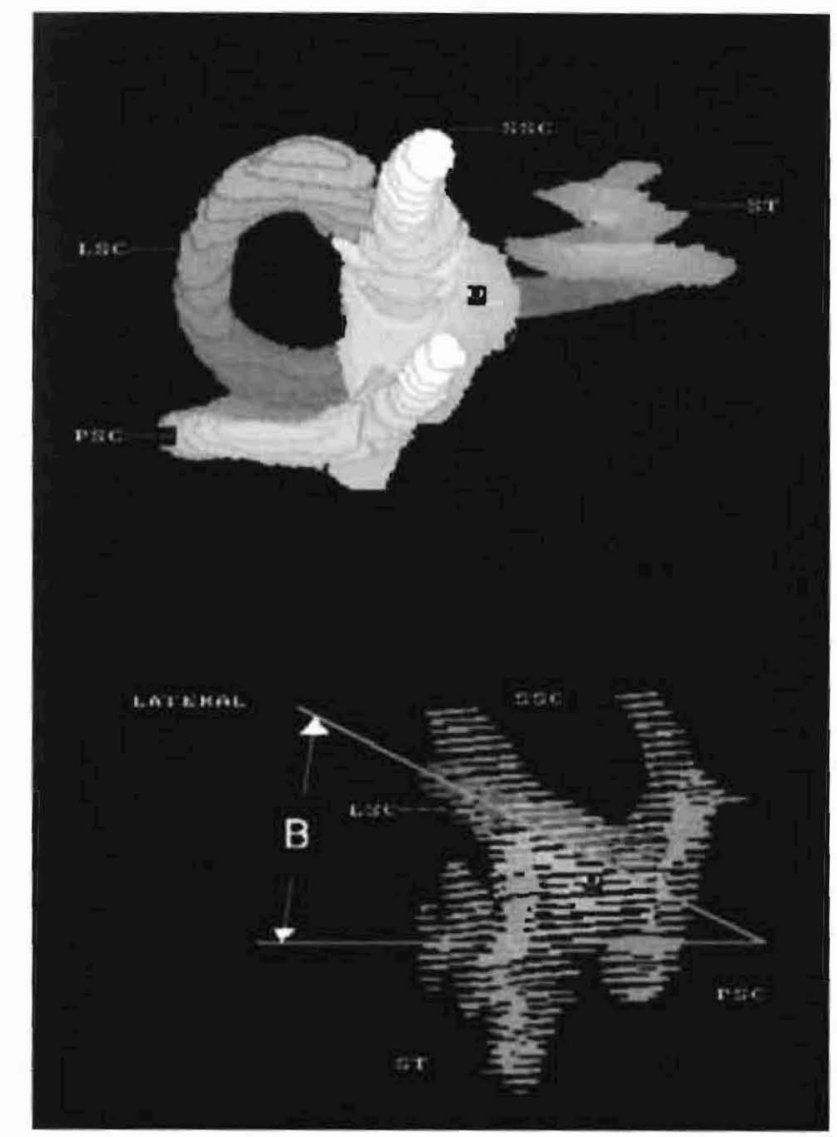

FIG. 2. A computer-aided three-dimensional graphic reconstruction of the labyrinth from histologic sections of a human temporal bone, viewing from the vertex (above) and from the lateral side of the labyrinth (below). $\mathrm{V}=$ vestibule; $\mathrm{ST}=$ scala tympani; SSC = superior semicircular canal; LSC = lateral semicircular canal; $P S C=$ posterior semicircular; $B=$ angle between LSC and the axis of the cochlea. make Silastic (Dow Corning, Midland, MI) molds of the labyrinth. These temporal bones were fixed in absolute alcohol for $>24 \mathrm{~h}$ and then resected. The whole bony labyrinth was maintained intact. The oval window was opened, and the round window membrane was incised with a 30 -gauge needle. A small hole was drilled at the helicotrema. The bony labyrinth was dehydrated in absolute alcohol and dried in an oven at $37^{\circ} \mathrm{C}$. Dow Corning MDX-4-42 I0 Silastic rubber was chosen as the molding material. One part of the curing agent was thoroughly mixed with 10 parts of the base material by weight. The mixture was degassed under vacuum $(-100 \mathrm{kPa})$ for 15 min to remove any bubbles and then aspirated into a syringe, care being taken to avoid air bubbles. Silastic was gradually injected with minimal pressure into the labyrinth through the oval window until it leaked out through the hole at the helicotrema and the round window. The bony labyrinth containing Silastic was kept at room temperature for at least 3 days until the Silastic had cured. The bony wall of the labyrinth was trimmed away so that an intact Silastic mold could be removed from the labyrinth. Angle B (see Fig. 2, below) between the LSC and the axis of the cochlea was measured through an image analyzer. Determining angle $\mathrm{B}$ is important also because it presents the angle needed to adjust for the horizontal plane of the skull.

\section{RESULTS}

The results of the measurements from the CT images in the axial plane are shown in Figure 3. The angle A (see Fig. I B) between the posterior semicircular canal and the midsagittal line varied from $46^{\circ}$ to $61.5^{\circ}$, the mean value was $52.5^{\circ}$ over the entire population (see Fig. $3 \mathrm{~A}$ ), $54.59^{\circ}$ in the child group and $51.28^{\circ}$ in the adult group (Fig. 3 B). There was a significant difference in angle A when adult data were compared with those from children $(\mathrm{p}<0.001 ; t$ test $)$.

The difference between the two readings at the axial section containing the cochlea (the modiolus section) and the axial section containing the orifice of the external auditory meatus varied from $1 \mathrm{~mm}$ to $14 \mathrm{~mm}$. The mean value was $7.18 \mathrm{~mm}$ over the entire population, $6.14 \mathrm{~mm}$ in the child group, and $8.22 \mathrm{~mm}$ in adult group. There was a significant difference when adult data were compared with those from children $(p<0.01 ; t$ test $)$. The results showed that the cochlea is approximately superior to the orifice of the external auditory meatus by $0.72 \mathrm{~cm}$ $(\mathrm{SD}=0.29 \mathrm{~cm})$.

The results of the measurements from 3D reconstructed images from histologic sections and Silastic molds of 10 labyrinths in the vertical plane revealed that angle B (see Fig. 2, below) between the LSC and the axis of the cochlea varied from $24^{\circ}$ to $30^{\circ}\left(24^{\circ}, 28.5^{\circ}, 30^{\circ}\right.$, $28^{\circ}, 30^{\circ}, 25^{\circ}, 30^{\circ}, 28^{\circ}, 30^{\circ}, 28.5^{\circ}$ ); the average angle was $28.2^{\circ}$. It is well known that the LSC forms an angle of $30^{\circ}$ upward and forward to the horizontal plane of the skull (11). Therefore, the axis of the cochlea lies almost parallel to the horizontal plane of the skull.

On the basis of these results from measurements in two planes, an optimal skull radiographic projection was designed to show the multichannel intracochlear electrode array and the corresponding structures of the inner ear. We have named this the cochlear view. 


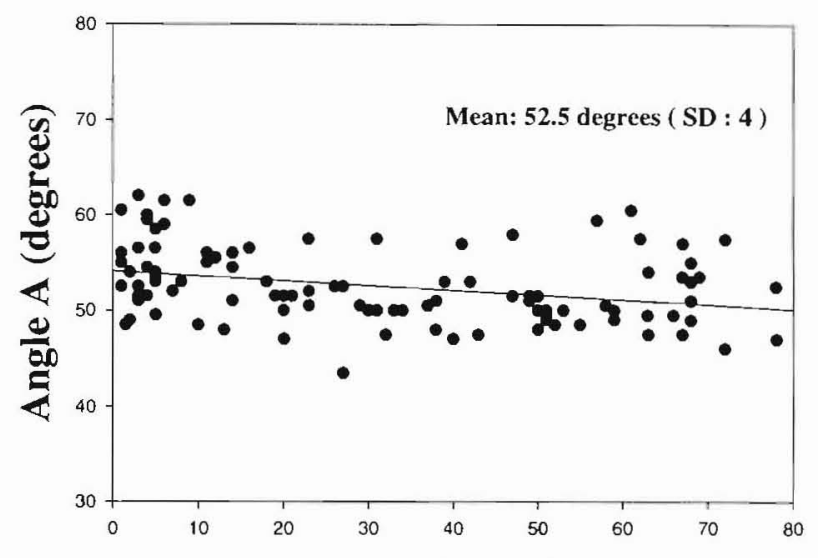

A

Age (years)

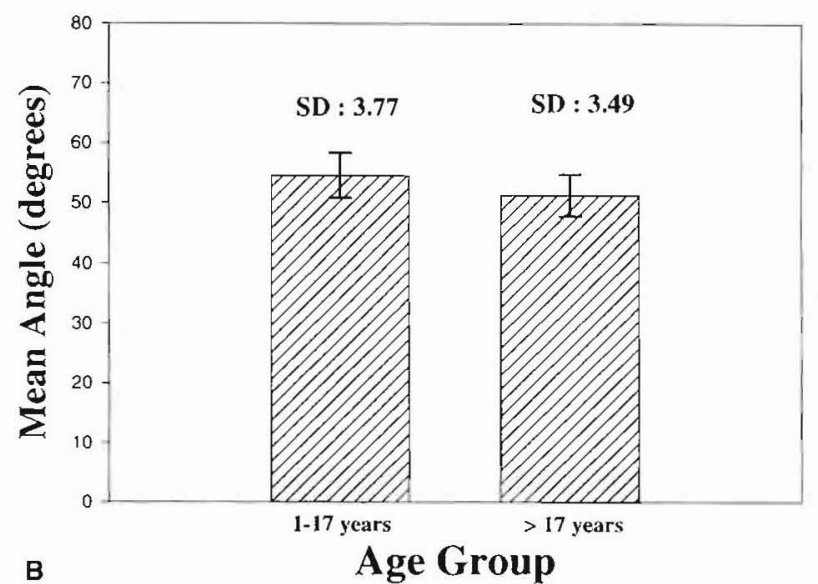

FIG. 3. Graph of measured angle A versus the age in 102 subjects (A), and mean values in the child group and the adult group (B).

\section{Radiography of cochlear view}

The patient is seated in front of a vertical device (Fig. $4 \mathrm{~A}$ ). The head rests on the forehead, nose, and zygomatic bone on the implanted side, and the median sagittal plane (M) is then adjusted to form an angle of $50^{\circ}$ with the plane of the film $(\mathrm{F})$, a protractor being used as a guide. The flexion of the neck is adjusted to make the horizontal plane (HP) containing two infraorbitomeatal lines (8) perpendicular to the image receptor (see Fig. 4 B). A fine-focus tube and a small localizing cone are essential (8). The central ray (CR) penetrates the occiput without any angulation and exits from the skull at a point $-3.0 \mathrm{~cm}$ anterior and $0.7 \mathrm{~cm}$ superior to the external auditory meatus closest to the image receptor.

Images are taken without a grid. The use of macroradiography, namely an enlargement technique, to show the microelectrode array is particularly rewarding. The focus-object distance is $90 \mathrm{~cm}$, and the object-image distance is $38 \mathrm{~cm}$ in our machine. An exposure of 80 $\mathrm{mAs}$ and $80 \mathrm{kV}$ was used for a medium-sized adult, with a fine-detail cassette.

\section{Resulting radiograph of cochlear view}

In a cochlear view radiograph (Fig. $5 \mathrm{~A}, \mathrm{~B}$ ), the vestibule $(\mathrm{V})$ is identified as an oval lucency, which is directly lateral to the IAM. Inferiorly, it is bordered by the most proximal extremity of the basal turn of the cochlea. Superiorly, the SSC appears as a vertical lucent line or elliptical ring surrounded by the dense bony capsule. The superior bony margin of the SSC forms a convexity on the roof of the petrous bone; this convexity is called the arcuate eminence. Laterally, the LSC is also seen as a horizontal lucent elliptical ring.

The bony capsule of the cochlea is visualized as an extremely dense shadow just at the bottom of the IAM. The multichannel intracochlear electrode array (E) appears as a two-dimensional spiral with its basal part located inferiorly. The IAM can be seen clearly as a lucent channel with well-defined walls. The round window is not visualized on a plain radiograph, but it would be inferior to the vestibule.

Radiologically, the SSC and the V can be easily recognized and form important landmarks in the radiologic anatomy of the cochlear view.

\section{APPLICATION OF THE COCHLEAR VIEW}

Patients receiving the Nucleus multichannel cochlear implant in our clinic are routinely evaluated postoperatively with the cochlear view. A single plain image of the cochlear view offers a complete and direct image of the intracochlear electrode array. The radiograph objectively documents the results of implant surgery, and any kink or postoperative slippage of the electrode array can be easily detected.

To evaluate objectively the depth of the intracochlear electrode array, fixed reference points should be used. We chose the apex of the SSC and the midpoint of the vestibule to draw a reference line (see Fig. 5 B, Fig. 6). To estimate the location of the round window in relation to the reference line, radiographs of nine human temporal bones were made with a Nucleus electrode array inserted in the scala tympani (12). The cochlear view of these bones indicated that the extension of the line drawn through the apex of the SSC and the midpoint of the vestibule reached the electrode at the inferior part of the round window.

The insertion depths of Nucleus electrode arrays from 106 patients were measured in our early studies (13 and $\mathrm{Xu}$ et al., unpublished data). The average insertion depth was $18.5 \mathrm{~mm}$ in length and $335^{\circ}$ in angle. However, we found no definite relationship between insertion depth in millimeters and insertion angle in degrees. This could be explained by the differences in cochlear size and the position of the electrode array, which could be close 10 the modiolus or the lateral wall.

An improved method for estimating the position of the electrodes of an implanted array using the cochlear view has been developed (12). The purpose of the method was to quantify angles for the individual bands of the array. To do this, it was necessary to estimate a center point of 

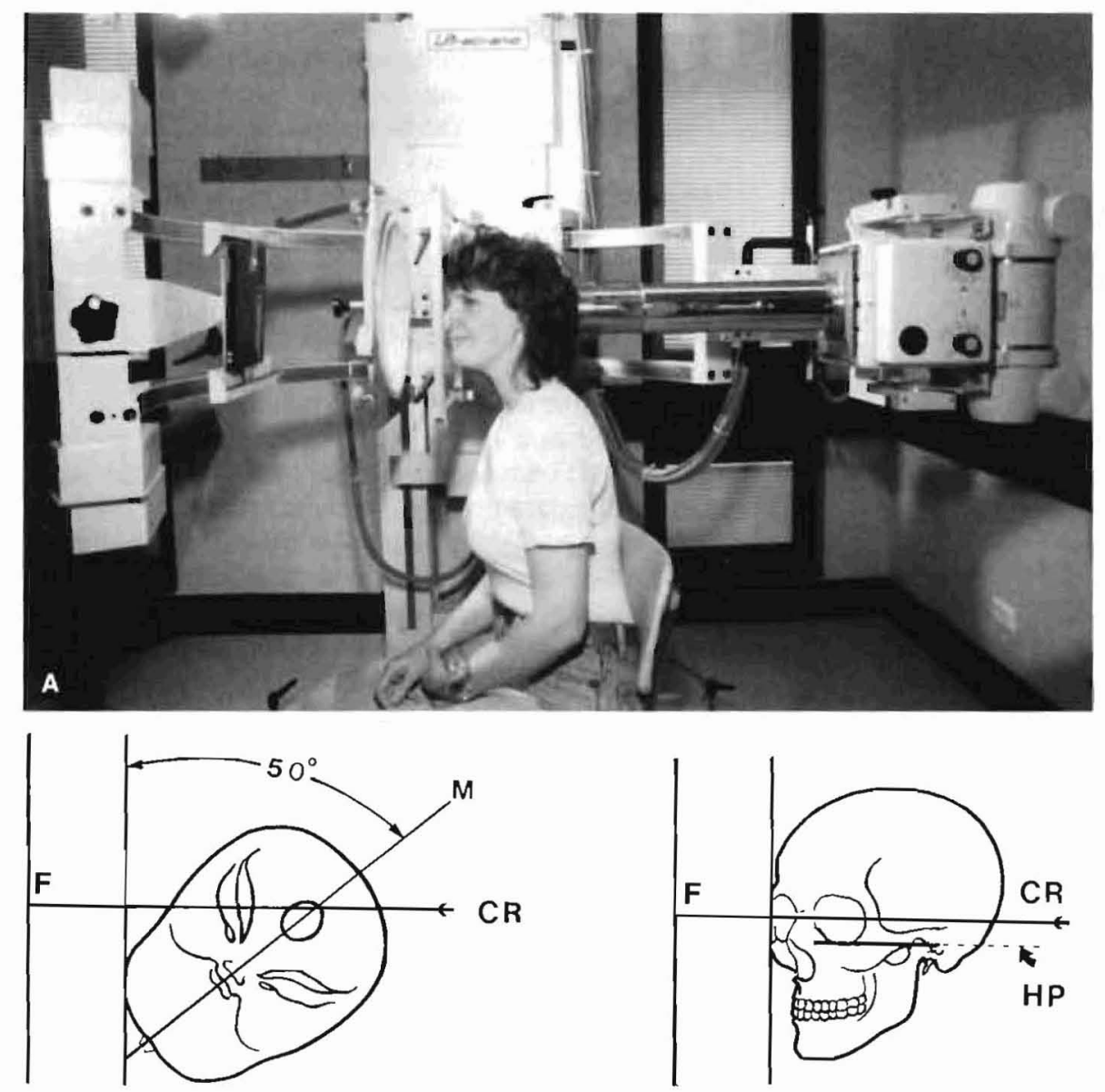

FIG. 4. A: Macroradiography of the cochlear view. B: Schematic diagram of macroradiography of the cochlear view. $C R=$ central $x$-ray beam; $F=$ image receptor; $\mathrm{M}=$ midsagittal plane of the skull; HP = horizontal plane (containing infraorbitomeatal lines). the cochlear spiral, about which the angles would be measured (see Fig. 6). A line drawn through that center point and at right angles to the reference line through the apex of the SSC and the midpoint of the vestibule would define the $0^{\circ}$ orientation. The center point was obtained, in the research method, by fitting a mathematical spiral to the positions of the bands of the array, as digitized on an image of the radiograph. The function for the mathematical spiral was determined from the cochlear view radiographs of 30 patients (12). When the spiral is fitted to the radiographic data, the center point and also the size of the template spiral are allowed to vary, because cochleas vary considerably in size. The spiral template function is described by the following expressions:

$$
\begin{gathered}
\theta \geq 100^{\circ}: \mathrm{R}=\mathrm{A} \exp (-\mathrm{B} \theta) \\
\theta<100^{\circ}: \mathrm{R}=\mathrm{C}\left[1-\mathrm{D} \log _{e}\left(\theta-\theta_{0}\right)\right]
\end{gathered}
$$

where $R$ is the radial distance from the spiral center, $\theta$ is the angle in degrees (see Fig. 6) and A, B, C, D, and $\theta_{0}$ are constants. The optimal value for B was 0.001317 . The corresponding mean value for $\mathrm{A}$, the size parameter, was $3.762 \mathrm{~mm}$. For male subjects, the mean value was $3.84 \mathrm{~mm}(\mathrm{SD}=0.19)$, and for female subjects it was $3.66 \mathrm{~mm}(\mathrm{SD}=0.20)$. The parameters $\mathrm{D}$ and $\theta_{0}$ $(0.12869$ and 5.0$)$ were chosen so that when the logarithmic function was made equal to the exponential function at $100^{\circ}$, the spiral would pass, on average, through a mean round window position. Once the mean template shape had been determined, $B, D$, and $\theta_{0}$ remained constant.

The clinical method did not require imaging equipment for digitizing the positions of the electrode bands. It did, however, still make use of the mathematical template spiral function just described. In this method, it is assumed that the array will follow a standard trajectory (a cursory inspection of the radiograph will reveal if it does not). There are only two relevant unknowns: the size of the spiral and the point at which the array crosses the reference line between SSC and V. The clinician counts the electrode bands, starting from the basal end of 


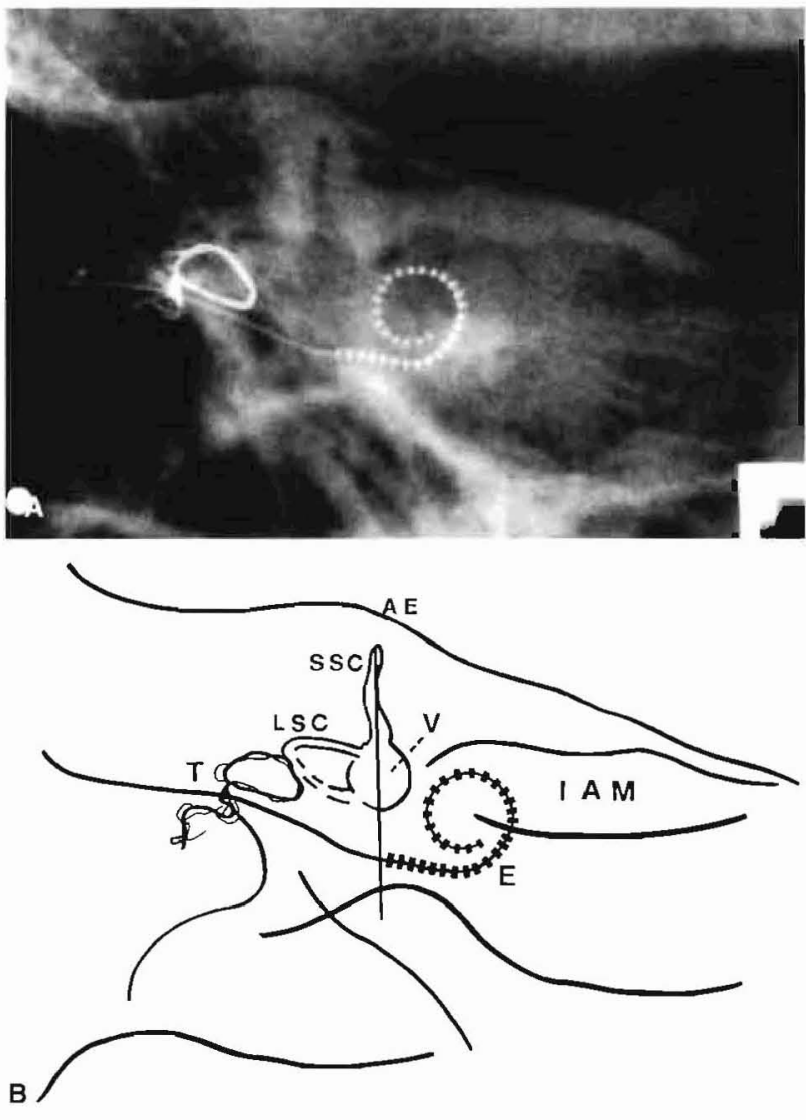

FIG. 5. A: Radiograph of the cochlear view from a cochlear implant patient. B: Schematic diagram drawn from (A). LSC = lateral semicircular canal; $\mathrm{SSC}=$ superior semicircular canal; $\mathrm{V}=$ vestibule; IAM = internal auditory meatus; $E=$ multichannel intracochlear electrode array; $T$ = fixation tie; $A E=$ arcuate eminence.

the array, and notes the count where the array crosses the reference line and the counts where it is tangential to lines either parallel to or at right angles to the reference line (see Fig. 6). A computer program uses the band counts to determine the size of the template spiral consistent with the data, and calculates angles for the individual bands of the array. The clinical method relies on the known and uniform spacing between the bands of the Nucleus array, whereas the research method need not be so constrained, provided the necessary calibralions are performed.

Clinically, surgeons and audiologists would need the cochlear view radiograph, a light box, and a transparency marked with the reference line and a grid (see Fig. 6). After the transparency is superimposed on the $x$-ray, and the reference line is aligned to pass through SSC and V, starting from the most basal stiffening band, the number on crossing the reference line (see 5.5 in Fig. 6) and the number at the most apical tangent point reached by the array (see 30.6 in Fig. 6) can be counted. These band counts are entered into a computer, which will calculate the spiral size automatically and estimate the ins stion angles for all the bands, as described earlier. The insertion length of the electrode array can be calculated easily by use of the following formula: number of bands in cochlea multiplied by the band separation $(0.75 \mathrm{~mm}$ center-center for the Nucleus electrode array).

Insertion angle data can be used to estimate characteristic frequencies corresponding to the bands of an implanted electrode array. The data of Bredberg (14) provide a relationship between angle and percentage length along the organ of Corti, while the expression of Greenwood (15) gives frequency as a function of fractional length along the organ of Corti. Therefore, frequency can be expressed as a function of angle. The zero angle of Bredberg ( 14 ) corresponded to the basal end of the organ of Corti. The data from our previous study (12) showed that the basal end of the organ of Corti- $0^{\circ}$ in Bredberg's (14) scheme-corresponded to an angle of $\sim 10^{\circ}$ in the present scheme. The resulting relationship between angle (in the present scheme) and characteristic frequency is plotted in Figure 7. This information can be used as a guide for programming the patient's speech processing strategies.

Radiographs obtained by he cochlear view are also used in other research projects in our department, includ-

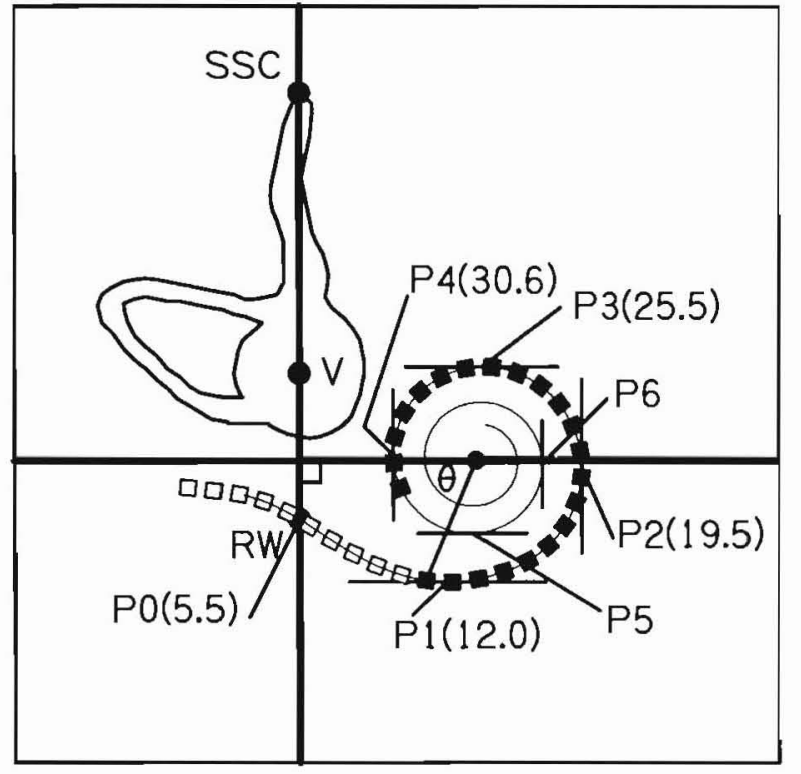

FIG. 6. Diagram showing the clinical method for estimating the insertion angles of each electrode from an implanted array using the cochlear view. Geometric construction involves drawing a reference line through the apex of the superior semicircular canal (SSC) and the midpoint of the vestibules (V), estimating the center of the electrode spiral, and constructing a line perpendicular to the reference line and passing through the spiral center. The electrode bands are counted starting with the most basal stiffening band $(\square)$. The counts are noted at the points shown, from point $O(P O)$ where the array cross the reference line to the most apical tangent point (P4 in this example). A computer is used to calculate the spiral size required to give these band counts, and estimates the insertion angles for all the electrodes. $\square=$ stiffening bands of electrode array; $\mathbf{\square}=$ conductive bands of electrode array; $\theta=$ resulting insertion angle. 


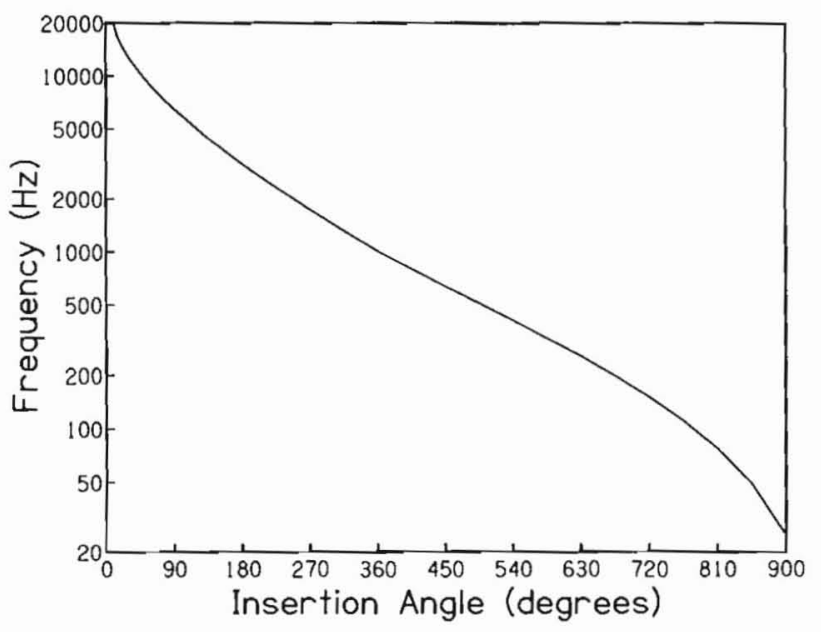

FIG. 7. Characteristic frequency versus insertion angle. In our method, a line drawn through the center point of the cochlear spiral and at right angles to the reference line defines the $0^{\circ}$ orientation (see Fig. 6).

ing the development of new cochlear implant electrodes designed to lie closer to the modiolus than the array currently in use $(16,17)$. Data from cochlear view radiographs are analyzed to evaluate the positions of the individual bands of developmental arrays in temporal bones, particularly the distance from the modiolus. Figure 8 shows the position of a prototype precurved array in a temporal bone, compared with that of a standard straight array in a patient.

\section{DISCUSSION}

To achieve optimal results from the cochlear view, as described in the section on the resulting radiograph, the following considerations are important:

1. The principle of designing the cochlear view was to make the $x$-ray beam direct to the cochlea and parallel to the axis of cochlea (see Fig. I B). The angle $\mathrm{A}^{\prime}$ between the sagittal plane of the patient's head and the image receptor we established for the cochlear view is appropriate for the majority of heads. Patients with an atypical cranium shape may require adjustment of the head position to obtain an ideal image. In general, patients with a brachycephalic (short front-to-back) skull require an increase in angle $\mathrm{A}^{\prime}$ (see Fig. I B and Fig. 4 B) because of a wider angle A (see Fig. I B) compared with the normal-shaped skull, and patients with a dolichocephalic (long front-to-back) skull require a decrease in angle $\mathrm{A}^{\prime}$ because of a narrower angle $\mathrm{A}$ compared with the normal-shaped skull. Also, the mean value of angle $A$ in the child group was slightly larger than in the adult group. Angle A can be measured in a preoperative CT image. Our previous study (13) showed that a $10^{\circ}$ difference in head positioning did not significantly alter the depth of insertion, as measured in degrees or mil- limeters. Therefore, only if the image is distortedfor example, the electrode array appears as a tilted two-dimensional spiral, or the SSC and V cannot be recognized - is repositioning of the patient's head necessary.

2. The contrast between the dense bony structure and the fluid-filled membranous structure provides the possibility of highlighting several landmarks on a plain radiograph, such as the vestibule and the semicircular canals. These landmarks are very important in determining depth of insertion (length and angle) and position of the individual electrode. In addition to proper head positioning, suitable exposures are important. The exposures (kilovolts and milliampere-seconds) used in our clinic are

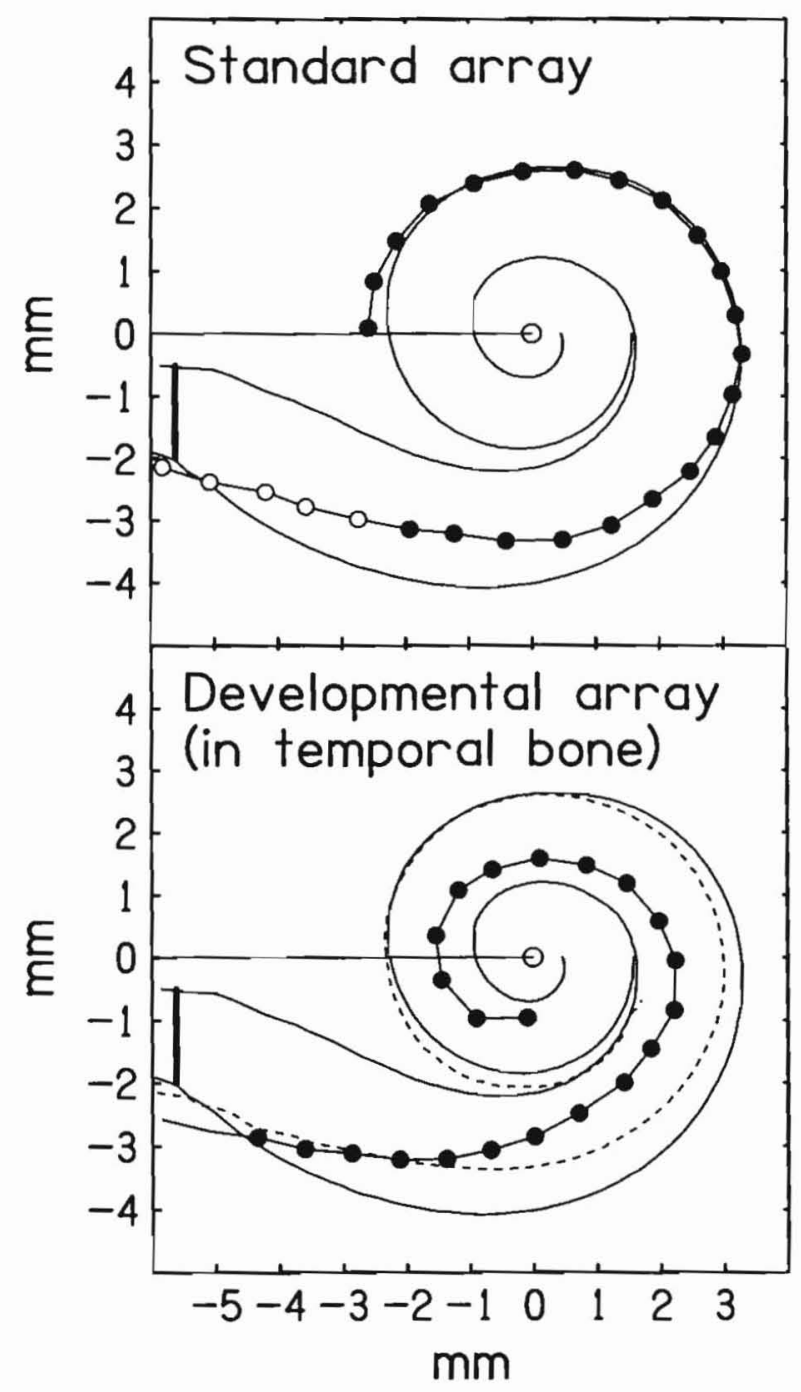

FIG. 8. Reconstructed diagrams from the cochlear view showing the position of a prototype precurved electrode array in the scala tympani of a temporal bone, compared with the position of a standard straight array in a patient. Solid line = mean position of outer and inner walls of scala tympani; dashed line = mean position of the standard straight array. 
only indications for other centers; exposures may need to be adjusted to obtain optimal contrast.

3. Macroradiography is vital to show the microelectrode array. If macroradiographic facilities are not available, the image-object distance can be increased to achieve a similar effect.

New computer algorithms for submillimeter resolution and 3D reconstruction from spiral computed tomographic scans show great promise for accurate determination of the position of implanted electrodes within the cochlea (4), but the individual electrodes cannot be distinguished (5). The plain radiograph cochlear view is a good alternative, giving a clear image of the whole intracochlear electrode array and each individual electrode. Its simplicity, minimal radiation, and low cost make the cochlear view useful and practical. It can be widely used in all cochlear implant clinics and research institutes.

The cochlear view has become a routine radiographic procedure for postoperative assessment of cochlear implantation in our clinic. It is an objective documentation of the depth of electrode insertion. In most cases, surgeons can demonstrate the success of the insertion from the cochlear view. In some cases in our clinic, patient performance was not consistent with the surgical estimate of insertion depth. It was found from the cochlear view that the electrode array was kinked in the proximity of the basal turn.

The research and clinical methods (12) were developed to extract quantitative information from cochlear view radiographs. Audiologists and speech pathologists have found the clinical method (12) for specifying the position of each electrode useful for managing frequency mapping and optimizing speech processing strategies. The research method, combined with computer analysis, can provide information about the lateral position of the array within the scala tympani, which is very useful for the development of improved electrode arrays (16,17).

Cochlear view provides a primary source of information, which can be analyzed in many ways.

\section{CONCLUSION}

On the basis of anatomical studies, the cochlear view technique of skull radiography was developed. Application of the cochlear view has shown that it is a useful postoperative procedure for documenting the results of implant surgery and for evaluating the depth of insertion (length and angle) and position of the individual electrode bands. It serves as a valuable reference for managing frequency mapping, optimizing speech processing strategies, and conducting research studies. The method can be widely used in all cochlear implant clinics because of its simplicity, low radiation, speed, and minimal cost.

Acknowledgment: The authors thank Julianna M. Silverman for radiographic assistance, Dr. Lee Seldon for providing the 3D reconstruction technique, and Dr. Robert K. Shepherd for revising the manuscript. Computed tomography images were provided by the Royal Victorian Eye and Ear Hospital and the Royal Children's Hospital in Melbourne, Australia.

\section{REFERENCES}

1. Beaumont D, Whyte A. Radiology of the tesnporal bonc. Aust J Otolaryngol 1994:1:513-26.

2. Rosenberg RA, Cohn NL, Reede DL. Radiographic imaging for the cochlear implant. Ann Otol Rhinol Larvngol 1987:96:300-4.

3. Gray RF, Evans RA, Freer CEL, Szutowič HE, Maskell GF. Radiology for cochlear implants. J Laingol Otol 1991:106:85-8.

4. Skinner MW, Ketten DR, Vannier MW, Gates GA. Yoffie RL, Kalender WA. Determination of the position of Nucleus cochlear implant electrodes in the inner ear. Am J Otol 1994: I 5:644-51.

5. Himi T, Kataura A, Sakata M, Odawara Y, Satoh J. Sawaishi M Three-dimensional imaging of the temporal bone using a helical $\mathrm{C} T$ scan and its application in patients with cochlear implantation. J Onol Rhino Larvingol Rel Spec 1996;58:298-300.

6. Portnoy WM. Mattucci K. Cochlear implants as a contraindication to magnetic resonance imaging. Ann Otol Rhinol Laryngol 1991: 100: 195-7.

7. Hasso AN Vienaud J, de Smedi E. Normal anatomy of the temporal bone and mastoid. In: Newton TH. Hasso AN. Dillon WP. eds. Compuled Tomography of Head and Neck. New York: Raven Press. 1988.

8. Ballinger PW: Merrill's Allas of Radiographic Positions and Radiologic Procedure, 8th ed. St. Louis: CV Mosby, 1995.

9. Sato H. Sando I. Takahashi H. Fujita S. Torsion of the human semicircular canals and its influence on their angular relationship. Acta Orolaryngol (Stockh) 1993;113:171-5.

10. Seldon L. Three-dimensional reconstruclion of temporal bone liom computed tomographic scans on a personal computer. Arch Onolaryngol Hoad Neck Surg 1991:117:1158-61.

II. Correia MJ, Dickınan JD. Peripheral vestibular system. In: Paparella MM. Shumrick DA. Otolaryngology, 3rd ed. Vol. 1. Philadelphia: WB Saunders, 1991:269.

12. Cohen LT. Xu J, Xu SA, Clark GM. Improved and simplified methods for specifying the position of the electrode bands of a cochlear implant array. Am J Orol 1996;17:859-65.

13. Marsh MA, Xu J, Blamey PJ, el al. Radiological evaluation of multiple-channel intracochlear implant insertion depth. Am $J$ Orol 1993;14:386-91

14. Bredberg G. Cellular pattern and nerve supply of the human organ of corti. Acta Otolaryngol (Stockh) 1968;(suppl 236): $1-138$.

15. Greenwood DD. A cochlear frequency-position function for several species-29 years later. J Acous Soc Am 1990;87:2592-605.

16. Shepherd R, Treaba C, Cohen L, et al. Peri-modiolar electrode arrays: a comparison of electrode position in the human temporal bone. XVI World Congress of Otorhinolaryngol Head and Neck Surg, Sydney, Australia. 1997

17. Tykocinski $M$, et al. Comparison of electrode position in the human cochlea using various peri-modiolar electrode arrays. Am J Otol (in press). 


\section{University Library}

\section{- M M N E R VA A gateway to Melbourne's research publications}

Minerva Access is the Institutional Repository of The University of Melbourne

Author/s:

XU, JIN;Xu, Shi-Ang;Cohen, Lawrence T.;Clark, Graeme M.

Title:

Cochlear view: postoperative radiography for cochlear implantation

Date:

2000

Citation:

Xu, J., Xu, S., Lawrence, C. T., \& Clark, G. M. (2000). Cochlear view: postoperative radiography for cochlear implantation. American Journal of Otology, 21(1), 49-56.

Persistent Link:

http://hdl.handle.net/11343/27581 